\title{
Explicación sin invarianza. El caso de los procesos socioeconómicos
}

\author{
EXPLANATION WITHOUT INVARIANCE. THE CASE OF SOCIOECONOMIC PROCESSES
}

Dr. Leonardo Ivarola (ivarola@economicas.uba.ar) Facultad de Ciencias Económicas, Universidad de Buenos Aires (Buenos Aires, Argentina)

\begin{abstract}
The main models of scientific explanation assume the need for some kind of stable knowledge (laws, invariant regularities, mechanisms, etc.) for assembling a good explanatory argument. While these approaches are useful in the natural sciences, it is doubtful that they are similarly applicable in the socioeconomic realm. In this paper it is expected to show that the logic of socioeconomic processes of being "possibility trees" or "open-ended results" makes regularities the exception rather than the rule. Alternatively, a mode of explanation that focuses on contextual factors and people actions is proposed.
\end{abstract}

Keywords: explanation, invariance, mechanisms, socioeconomic processes, possibility trees.

\section{Resumen}

Los principales modelos de explicación científica asumen la necesidad de utilizar algún tipo de conocimiento estable (leyes, regularidades invariantes, mecanismos, etc.) para el armado de un buen argumento explicativo. Si bien estos enfoques son muy útiles en las ciencias naturales, es dudoso que sean similarmente aplicables en el reino de lo socioeconómico. En el presente trabajo se prevé mostrar que la lógica de los "árboles de posibilidades" o "resultados de final abierto" propias de los procesos socioeconómicos hace que las regularidades sean más la excepción que la regla. Como alternativa, se propone un modo de explicación que focalice en los factores contextuales y en las acciones de las personas en el momento en que aconteció el fenómeno a explicar.

Palabras clave: explicación, invarianza, mecanismos, procesos socioeconómicos, árboles de posibilidades.

\section{Introducción}

Explicar un fenómeno X implica responder a la pregunta “¿por qué X?” o “¿cómo se produce X?”. El enfoque estándar de explicación científica es el modelo de cobertura legal desarrollado por Hempel y Oppenheim (1948). Según este enfoque, para que una explicación sea considerada "científica", la misma debe incluir, al menos, una ley. Éste es el modo en el cual la explicación se diferencia del mero descriptivismo. Así, decimos que un metal $\mathrm{X}$ se dilata con el calor porque existe una ley física por la cual todos los metales se dilatan con el calor, o decimos que lo que tarda en caer una bola de acero ubicada a 84 metros de distancia será aproximadamente de 4,14 segundos, puesto que existe una ley que da cuenta de dicho fenómeno. 
Sin embargo, fuera de la física y de la astronomía, el descubrimiento de leyes es un asunto sumamente complejo. Asimismo, en numerosas ocasiones las explicaciones basadas en leyes no son relevantes para el fenómeno que se trata de comprender. Para peor, asumiendo que no existen problemas ni de relevancia explicativa ni con el descubrimiento de leyes, el modelo de cobertura legal ha sido fuertemente criticado por proporcionar explicaciones de caja negra: se conoce el qué de un fenómeno, pero no el cómo.

Diferentes alternativas a este modelo de explicación científica se han propuesto a lo largo del tiempo. Woodward y Hitchcock (2003), por ejemplo, aseveran que las regularidades que forman parte del explanans -o argumento explicativo- deberían ser capaces de mostrar de qué depende el fenómeno a explicar, lo cual se logra en tanto dichas regularidades justifiquen contrafácticos "intervencionistas". Otros autores de corte "mecanicista" consideran que una buena explicación consiste en describir el mecanismo que da cuenta de un fenómeno o un patrón de conducta observado.

Ahora bien, un problema recurrente de los modelos de explicación científica es que éstos están pensados para las ciencias naturales. En estos sistemas predomina la invarianza, o, al menos, la estabilidad contributiva de buena parte de los factores causales bajo estudio. Esto es más controvertido en las ciencias sociales en general y en la economía en particular. Los fenómenos socioeconómicos son más complejos que los de la naturaleza. Las relaciones causales están mediadas por la acción humana, la cual no solamente es vulnerable a la información que recibe del mundo, sino también a las condiciones contextuales que propician o inhabilitan su conducta. Esta notoria inestabilidad hace que, en los sistemas socioeconómicos, las regularidades sean más la excepción que la regla.

Es erróneo pensar que sin regularidades y/o mecanismos no sea posible brindar una explicación que sea profunda -y hasta "científica". Sin embargo, los principales enfoques son explícitos en establecer que sin éstos no hay explicación que sea valiosa. En este marco, en el presente trabajo se procurará mostrar que ni las regularidades ni los mecanismos son necesarios para establecer una buena explicación. En una primera instancia se prevé mostrar -tal vez muy escuetamente- que los procesos socioeconómicos responden a una lógica de "árboles de posibilidades" o "resultados de final abierto", en lugar de una lógica mecanicista, propia de los sistemas de la naturaleza. Asociado a esto, se ofrecerá una salida alternativa a los principales modelos explicativos, mostrando que, en realidad, los factores del contexto y las acciones de las personas en el momento en que acontece el fenómeno a explicar juegan un rol más preponderante en el armado de un buen argumento explicativo, que la mera apelación a regularidades (sean tanto invariantes como legaliformes) y/o mecanismos.

\section{La explicación basada en regularidades}

El modelo de cobertura legal desarrollado por Carl Hempel y Paul Oppenheim en 1948 es considerado dentro de la filosofía de la ciencia como el modelo "clásico" de explicación científica. El término legal alude a la idea de que proporcionar una explicación acerca de un hecho consiste, fundamentalmente, en mostrar que éste obedece a leyes. No obstante, el fenómeno que se pretende explicar no sólo es una consecuencia de la operación de una o varias leyes, sino también de un conjunto de condiciones antecedentes. En este sentido, los autores diferencian dos partes fundamentales en el modelo: el explanans y el explanandum. El explanandum es un enunciado que describe un fenómeno empírico a explicar, el cual puede ser tanto un acontecimiento que ocurrirá en un lugar y tiempo determinado, como expresar una regularidad legaliforme. Por su parte, el explanans es el argumento explicativo, esto es, el fundamento de la ocurrencia del explanandum. Dentro del explanans se pueden diferenciar dos 
tipos de enunciados: (1) leyes universales y/o estadísticas (se establece como condición sine-qua-non que debe haber al menos una ley), y (2) condiciones antecedentes o hechos concretos. Se asume que estas dos clases de enunciados permiten inferir -sea deductiva como inductivamente- el enunciado explanandum.

Siguiendo a Hempel, para que una explicación pueda ser considerada científica, deben cumplirse ciertos requisitos, a saber, el de relevancia explicativa y el de contrastabilidad. Con respecto al primer requisito, el argumento propuesto debe proporcionar una buena base para creer que el fenómeno que se trata de explicar tuvo o tiene lugar. Con este requisito Hempel trata de dejar de lado las explicaciones espurias. En lo referente al requisito de contrastabilidad, los enunciados que constituyen una explicación deben ser susceptibles de contrastación empírica, esto es, deben poseer contenido empírico.

Aparentemente, el modelo de cobertura legal satisfaría estas dos condiciones: puesto que el enunciado explanandum es inferido del explanans, la información de este último proporciona una buena base para esperar que se dé el fenómeno explanandum. En segundo lugar, el explanandum tiene contenido empírico, y puesto que es consecuencia lógica del explanans, luego este último es contrastable indirectamente.

Al sólo efecto ilustrativo, tomemos como ejemplo un caso histórico citado por el propio Hempel: "En el otoño de 1946 se produjo en los mercados algodoneros de los Estados Unidos una baja tan aguda del precio, que los mercados de Nueva York, Nueva Orleans y Chicago tuvieron que suspender sus actividades transitoriamente. En la tentativa de explicar este hecho, la prensa norteamericana lo investigó hasta descubrir que un especulador en gran escala de Nueva Orleans, temiendo que sus acopios fuesen excesivos, había comenzado a venderlos; los especuladores menores, presos del pánico, habían seguido su ejemplo, con lo cual precipitaron la crisis" (1979:253).

De acuerdo con Hempel, este argumento es una buena explicación, ya que implica enunciados sobre condiciones antecedentes y el supuesto de regularidades generales. Las primeras incluyen los hechos de que el primer especulador tenía mucha producción de algodón, que había especuladores menores con considerables acopios del material, que existían instituciones tales como los mercados algodoneros con su modo específico de operar, etc. Las regularidades generales referidas no se mencionan explícitamente, pero hay una implicación obvia de alguna forma de la ley de la oferta y la demanda que explica la baja de los precios del algodón en función del gran aumento de la oferta con una demanda prácticamente estable. También es necesario contar con ciertas regularidades en la conducta de los individuos que tratan de mantener o mejorar su posición económica.

El modelo de cobertura legal ha sido cuestionado desde diferentes perspectivas. Una de las primeras críticas fue proporcionada por Wesley Salmon, quien se encargó de mostrar que el requisito de relevancia explicativa no logra expresar las condiciones necesarias y suficientes para una buena explicación. He aquí dos ejemplos de ello:

Ejemplo 1:

Juan confiaba en recuperarse de su resfrío porque tomaba vitamina $\mathrm{C}$, y casi todos los resfríos se curan dentro de una semana de tomar vitamina $C$. 
En el ejemplo se satisfacen los requisitos propuestos por Hempel, a saber, existe una ley (en este caso, estadística) dentro del explanans que asevera que "casi todos los resfríos se curan dentro de una semana de tomar vitamina C". Asimismo, existe un hecho concreto dentro del explanans: Juan ha estado tomando vitamina $\mathrm{C}$ durante varios días. El explanandum, además de ser observable, se infiere (inductivamente) del explanans: Existen altas chances de que se recupere (o confía en que se recuperará).

No obstante, Salmon apela al conocimiento científico para afirmar que los resfríos se curan en el plazo de una semana, independientemente de que tome o no vitamina C. De este modo, a pesar de cumplir formalmente con los requisitos exigidos por Hempel, la explicación es irrelevante. El segundo ejemplo es aún más evidente.

Ejemplo 2:

(I) Todos los hombres que toman regularmente pastillas anticonceptivas no quedan embarazados.

(II) Juan es una persona que ha estado tomando regularmente pastillas anticonceptivas.

(III) Juan no quedará embarazado.

Es trivial decir que independientemente de que tome las píldoras anti-conceptivas o no Juan no quedará embarazado. No obstante, el ejemplo anterior se adecúa perfectamente al modelo de cobertura legal, en función de que existe una ley (I), un hecho concreto (II), y un explanandum (III) que se infiere (en este caso) deductivamente del explanans. Así, a pesar de cumplirse con los requisitos demandados por Hempel, la explicación puede ser irrelevante.

Adicionalmente, un problema que Woodward y Hitchcock (2003) encuentran en el modelo de cobertura legal -y en general en cualquier modelo explicativo basado en leyes- es que éstas no constituyen un buen medio para conformar argumentos explicativos satisfactorios, ya que no siempre logran responder como cambiaría el fenómeno explanandum ante cambios en las condiciones de inicio. De acuerdo con el modelo de cobertura legal, una explicación es más profunda en la medida en que se apelen a regularidades cada vez más generales, esto es, que su dominio o rango de aplicabilidad sea lo más grande posible. Woodward y Hitchcock, por el contrario, aseveran que este enfoque focaliza en la clase equivocada de generalidad: generalidad respecto de objetos o sistemas. La clase correcta de generalidad es aquella asociada con la exhibición de patrones de dependencia contrafáctica que describen cómo el sistema cuyo comportamiento deseamos explicar cambiaría ante diferentes condiciones.

De acuerdo con Woodward y Hitchcock, explicar científicamente un fenómeno consiste en mostrar de qué depende el mismo. Las leyes no proporcionan ese tipo de conocimiento. Sólo permiten inferir un resultado, pero no mostrar de qué depende. Un enfoque basado en leyes apunta a responder preguntas del tipo "¿por qué $x$ ?". No obstante, Woodward y Hitchcock consideran que los argumentos que efectivamente proporcionan profundidad explicativa son aquellos que permiten responder al rango de preguntas "¿qué pasaría si las cosas fueran diferentes?", pregunta que terminaría clarificando la dependencia causal del fenómeno explanandum.

En este marco, consideremos la pregunta "¿qué pasaría si Juan no tomara las pastillas anticonceptivas?". Si el resultado "embarazo" dependiese de la ingesta de pastillas, se esperaría observar un resultado diferente. Pero esto no es así. El fenómeno explanandum no depende del consumo de anticonceptivos. 
Por consiguiente, este argumento -aunque basado en "leyes"- fracasa en proporcionar una base explicativa coherente.

Woodward y Hitchcock consideran que para responder adecuadamente a la pregunta "¿qué pasaría si las cosas fuesen diferentes?" no se necesitan leyes, sino regularidades que sean invariantes bajo intervenciones en las variables independientes. Estos dos conceptos -invarianza e intervenciónnecesitan ser clarificados.

En primer lugar, hablar de invarianza implica hablar de estabilidad o de alta correlación entre variables. Cuanto mayor sea el grado de invarianza, mayor será la profundidad explicativa de esa relación. Sin embargo, la invarianza per se no es suficiente para comprender de qué depende el fenómeno explanandum. Por el contrario, se necesita que la regularidad sea invariante bajo manipulaciones en la(s) variable(s) independiente(s).

Para entender este punto, consideremos el siguiente ejemplo. Sean $\mathrm{X}$ la altura de la columna de mercurio de un barómetro, $\mathrm{Y}$ el nivel de precipitaciones y $\mathrm{U}$ el término de error representando todos los factores causales omitidos. La regularidad puede expresarse de la siguiente manera:

$$
Y=F(X)+U
$$

Esta generalización describe el nivel de precipitaciones en función de la altura de la columna de mercurio de un barómetro. En principio, se requiere que esta relación se mantenga estable ante un cierto rango de cambios, no de todos. He aquí la diferencia con una ley. En el marco de una ley no puede haber excepciones. Una relación invariante, por el contrario, permite esta posibilidad.

Sin embargo, (1) se mantiene ante cambios en $X$ en función de la dependencia de ambas variables respecto de una causa común: la presión atmosférica. Para que sea explicativa, Woodward y Hitchcock consideran que una regularidad debe permanecer invariante ante otra clase de cambios: las manipulaciones o intervenciones en la variable independiente.

Una intervención es un proceso causal exógeno que produce el antecedente en cuestión. Heurísticamente, y sólo heurísticamente, las intervenciones pueden ser entendidas como manipulaciones llevadas a cabo por la acción humana en un experimento idealizado. En otras palabras, la noción de intervención que se propone aquí trata de capturar, en un lenguaje no antropomórfico que no haga referencia alguna a nociones como la de agencia humana, las condiciones que se deberían conocer en una manipulación ideal sobre una variable independiente, llevada a cabo con el propósito de determinar si ésta causará algún cambio en la variable dependiente. Dicha manipulación no debe estar asociada con otras causas que provoquen un cambio la variable dependiente, así como tampoco debe afectar a esta última a través de una ruta que excluya la variable a manipular.

Para entender mejor este punto retomemos el ejemplo de la ecuación (1). Nuestro interés radica en comprender qué pasaría si se interviniese físicamente (sea ya por la acción humana como por un proceso natural) sobre $X$ con el propósito de modificar el valor de $Y$. Claramente, interviniendo sobre la altura de la columna de mercurio (por ejemplo, por la aplicación de calor al barómetro), no va a llover más o menos. La fuente de dicho problema estriba en que, como se dijo anteriormente, ambas variables son producto de una causa común: la presión atmosférica (a la cual denominaremos como " $Z$ "). De ello se tiene que cambios en $Z$ conducirá a cambios tanto en $X$ como en $Y$. Si bien $Z$ actúa como variable 
interventora, resulta que manipulando $X$ a través de $Z$ no cuenta como intervención sobre $X$ para modificar el valor de $Y$ (ni viceversa), ya que dicha manipulación afecta a $Y$ a través de una ruta que excluye a X.

¿Cómo reconocer si una regularidad va a ser útil para propósitos explicativos? De acuerdo con Woodward y Hitchcock, ésta debe poder justificar enunciados condicionales contrafácticos de un tipo particular. No cualquier contrafáctico sirve para reconocer cuando una relación es causal o no. La relación entre la altura del barómetro y la aproximación de la tormenta justifica contrafácticos del tipo "si la altura del barómetro disminuyese, la tormenta se estaría aproximando". Los contrafácticos requeridos en el enfoque manipulabilista de Woodward y Hitchcock son aquellos que involucran intervenciones hipotéticas: si se interviniese sobre $X$, modificando su valor, cambiaría el valor de $Y$. Woodward llama a estos contrafácticos "activos" o "intervencionistas".

La regularidad (1) no puede justificar esta clase de contrafácticos, ya que interviniendo adrede en la altura de la columna del barómetro (esto es, por medio de intervenciones producto de la agencia humana), no se va poder manipular el nivel de precipitaciones. Contrario a ello, tomemos como caso la relación entre la presión atmosférica y la columna del barómetro, y veamos si es posible justificar contrafácticos intervencionistas: (a) si se manipulase la columna del barómetro, esto no modificaría la presión atmosférica; (b) si se manipulase la presión atmosférica, la columna del barómetro cambiaría de tamaño.

Si bien las dos aserciones hacen referencia a una misma correlación de datos (y por tanto justifican una determinada clase de contrafácticos), es sólo la segunda la que justifica contrafácticos intervencionistas. Tal como se puede apreciar, esta regularidad permite responder al rango de preguntas "¿qué pasaría si las cosas fuesen diferentes?", por lo que muestra con éxito de qué depende el fenómeno explanandum.

\section{La explicación a través de mecanismos}

Las regularidades -sean invariantes o legaliformes (leyes)- proporcionan información al nivel de los eventos. No obstante, éstas no logran dar cuenta ni de las razones que las justifican, ni de las condiciones que podrían anularlas. Por tanto, su utilización en un modelo explicativo podría no ser del todo efectiva, puesto que no permite entender el proceso que va desde la variable independiente hasta la dependiente. En otras palabras, estas regularidades ofrecen explicaciones del tipo "caja negra": conocemos los inputs y outputs, pero no sabemos el proceso por el cual al utilizar determinados inputs obtendremos tales outputs. Conocemos el qué, pero no el cómo. A este respecto, es menester citar la distinción que hace Mario Bunge (2005) entre tres tipos de teorías o hipótesis científicas, categorizadas en función de su profundidad o potencia explicativa: (a) de caja negra, descriptivas o fenomenológicas, que responden a la pregunta "¿qué es esto?", (b) de caja gris o semifenomenológicas, que dan respuestas superficiales a preguntas del tipo “¿cómo funciona esto?" y (c) de caja translúcida o mecanísmicas, que responden detalladamente a preguntas del tipo “¿cómo funciona esto?".

Los modelos mencionados en el acápite anterior proporcionarían explicaciones de caja negra: muestran el grado de correlación entre dos variables -inputs y outputs-, pero no muestra el modo en que el output es producido a partir de un conjunto de inputs. La ley de gases ideales pertenece a la categoría de "caja negra", puesto que muestra la relación entre volumen, temperatura y presión de un gas, pero no explicita el modo en que el cambio en una variable contribuye al cambio en otra. 
Una hipótesis de caja gris es la que representa los interiores de sus referentes de manera esquemática. Los modelos de red de los sistemas sociales caen en esta categoría. Éstos representan la composición y la estructura de los sistemas sociales, y relacionan los niveles micro con los macro. Sin embargo, debido a que son estáticos, tales modelos no pueden dar cuenta del crecimiento o la decadencia y mucho menos de ningún proceso de cohesión o de descomposición.

Una hipótesis de caja translúcida explicita el mecanismo por el cual, dado determinados inputs, ocurren ciertos outputs. Una teoría de caja translúcida es explicativamente más profunda que el resto porque no dice sólo lo que ocurre, sino también qué es lo que hace que algo ocurra.

Por lo que se puede apreciar, en este último caso se defiende la idea de que la propia apelación a mecanismos permitiría llenar el "vacío explicativo" que existe entre los modelos basados en regularidades, ya que no solo muestran el resultado de introducir un input en un determinado sistema, sino también cómo se da todo ese proceso.

Los modelos de explicación mecanicistas rescatan la noción de comportamiento regular o estable, la cual es entendida en términos de robustez. Por ejemplo, Stuart Glennan (2002) propone una visión de mecanismos que se aleje del enfoque de entender a éstos como secuencias de eventos interconectados. Según esta postura, un mecanismo es una cadena de eventos relativos a un acontecimiento particular, como puede ser la consecución de hechos que desembocaron en la rotura de un jarrón luego de que un niño hiciera rebotar una pelota de tenis dentro de su casa. Glennan llama a esta clase de cadenas "secuencias frágiles": aquellas que involucran una consecución de eventos que muy rara vez pueda volver a repetirse. En particular, las condiciones que favorecieron que ese resultado (la rotura del jarrón) tenga lugar son muy restrictivas, en el sentido de que cualquier cambio -por pequeño que sea- puede modificar el resultado final. Por ejemplo, un cambio mínimo en la fuerza con que la pelota es dirigida puede hacer que ésta rebote en un lugar diferente al inicial, haciendo que el curso de la pelota termine siendo diferente. En contraposición al concepto de secuencia frágil, Glennan define como "secuencia robusta" a los procesos que pueden repetirse en variadas ocasiones. Esta robustez es observable en el comportamiento de los mecanismos.

Sin embargo, como ya se ha comentado anteriormente, la invarianza o robustez de una relación no es suficiente para proporcionar un argumento con capacidad explicativa. Por ello se apela a los mecanismos: para entender el cómo de dicha relación. En general, la invarianza o robustez de un mecanismo es pensada dentro de este enfoque como una consecuencia de la solidez estructural del propio mecanismo, la cual a su vez es producto del comportamiento estable de las partes que lo constituyen. En palabras de Glennan: "mechanisms (...) are systems consisting of relatively stable configurations of parts that give rise to robust behaviors which can be expressed by invariant generalizations" (2002:348).

Tomemos como ejemplo las máquinas expendedoras de gaseosas o golosinas. Existe un proceso robusto o invariante, que comienza cuando se inserta la moneda y se oprime el botón correspondiente al producto deseado. En el interior de la máquina tienen lugar una serie de interrelaciones entre las partes, que culminan con la obtención del producto seleccionado. Sin embargo, la secuencia regular a la que se hace mención no está garantizada -entre otras cosas- en la medida en que no funcionen adecuadamente las partes que se encuentran en el interior de la máquina, y en tanto ésta no se encuentre adecuadamente aislada de un conjunto de factores que pudieran obstaculizar su correcto funcionamiento. 
¿Cómo es una explicación a través de mecanismos? Básicamente, ésta consiste en describir el mecanismo que justifica la aparición del fenómeno explanandum. Siguiendo a Glennan (2002), esta descripción involucra dos partes esenciales: (1) una descripción del comportamiento del mecanismo y (2) una descripción del mecanismo que da cuenta de ese comportamiento. La primera parte se refiere a la descripción externa del mecanismo, esto es, que es lo que "hace" un sistema, mientras que la segunda se refiere a la descripción interna del mecanismo, mostrando el "cómo" del funcionamiento de dicho sistema. Por ejemplo, el movimiento de las agujas del reloj no es otra cosa que el comportamiento externo del mecanismo "reloj", mientras que el modo en que las agujas se mueven -a través de distintos engranajes- muestra o describe el funcionamiento del propio mecanismo.

La descripción (2) constituye el explanans o argumento explicativo, mientras que (1) es el explanandum o evento a explicar. Sin embargo, hay una diferencia importante con los modelos basados en regularidades, y es justamente respecto de la conexión entre explanans y explanandum. En el modelo de cobertura legal, el explanandum se infiere sobre la base del explanans: " $X$ se dilata con el calor, ya que $X$ es metal, y todos los metales se dilatan con el calor". Incluso en el enfoque de Woodward y Hitchcock, donde se asevera que un buen argumento explicativo debe responder al rango de preguntas "¿qué pasaría si las cosas fueran diferentes?" o “¿cómo cambiará el explanandum ante un cambio en el explanans?", también se supone una conexión entre éstos mediada por una inferencia deductiva. Supongamos la ecuación $Y=2 X$. Ésta tendrá capacidad explicativa en tanto intervenciones en $X$ conduzcan a cambios proporcionales en $Y$. Sin embargo, el resultado de $Y$ se inferirá sobre la base de esta ecuación y de los valores que tome $X$. "Dada la ecuación $Y=2 X$, si $X=2$, entonces $Y=4$ ". Contrario a estos casos, la explicación mecanísmica supone una relación causal entre explanans y explanandum. Tomemos como ejemplo el efecto que causa el éxtasis sobre el organismo: "la ingesta de éxtasis causa aumento de serotonina, lo cual causa euforia". El fenómeno explanandum (euforia) no se infiere del explanans. No es producto de una relación lógica, sino causal.

\section{La explicación en los procesos socioeconómicos}

Hasta ahora se han examinado tres modelos explicativos: uno de ellos requiere de leyes, otro de regularidades invariantes bajo intervenciones en las variables independientes, y otro de mecanismos. No obstante, es cuestionable que dichos enfoques tengan la misma validez en sistemas donde las leyes, la invarianza y los mecanismos son la excepción más que la regla. Éste es el caso de los sistemas en donde tienen lugar los procesos socioeconómicos.

No se pretende dar aquí una definición de lo que es un proceso socioeconómico. Simplemente se lo entenderá como una secuencia de eventos que tuvo o tiene lugar en el mundo real. Esto significa que dichos procesos no son aproximaciones o representaciones de la realidad, sino que constituyen la realidad misma en un determinado momento y lugar.

A un nivel muy general, se puede decir que los procesos socioeconómicos se componen de dos elementos fundamentales: 1) las condiciones del contexto, que acotan el marco de acción de las personas y 2) las señales del mundo, que son interpretadas por los sujetos para tomar decisiones.

En principio, decir que los procesos sociales dependen de las señales del mundo significa decir que la información que los agentes reciben de éste $(v$. gr., cambios en variables económicas, anuncios políticos, tapa de un periódico, un rumor) constituye una señal a ser captada e interpretada por las personas. Estas interpretaciones conducirán a la formación de expectativas sobre estados del mundo futuro, el cual es 
incierto por definición. Finalmente, diremos que las personas actuarán sobre la base de sus interpretaciones y expectativas. Estas acciones darán lugar a nuevas señales, las cuales serán recibidas e interpretadas por otros agentes, etc.

Un ejemplo de un proceso cuyo resultado depende de las señales del mundo es la teoría de la profecía autocumplida formulada por Robert Merton. Ésta se refiere a un fenómeno social por el cual una señal informativa falsa tiene consecuencias verdaderas, las cuales eran inverosímiles antes del envío de dicha información. A modo de ejemplo, supongamos que se difunde el rumor sobre el posible quiebre de un banco. Algunos de los depositantes retirarán sus depósitos, haciendo que las reservas del mismo disminuyan. Esto reforzará aún más el rumor, por lo cual un número creciente de personas retirarán sus depósitos. Este proceso continuará hasta que el banco se quede sin reservas y termine finalmente presentando la bancarrota.

Asimismo, los procesos socioeconómicos dependen fuertemente de las condiciones del entorno, contexto o macro-estructura, las cuales "dan forma" al comportamiento de los individuos. Dicha estructura no es otra cosa que el conjunto de condiciones habilitantes e in-habilitantes de la acción humana. Esto significa que existen ciertas circunstancias del contexto que habilitan a las personas a tomar determinadas decisiones, así como también hay otras que las limitan. El proceso de la "bicicleta financiera" durante la década del 70' en Argentina es un buen ejemplo de ello: "El mecanismo utilizado era muy simple: se introducían dólares prestados por bancos extranjeros, se los pasaba a pesos, se realizaban ganancias por la tasa de interés nominal interna mucho mayor que el costo del crédito, fijado por la tasa de interés externa y el ritmo de devaluación, después se reconvertían los pesos a dólares, se los sacaba del país y se los depositaba en un banco extranjero, y se obtenía un nuevo crédito en dólares de ese banco con la garantía del depósito; y así se repetía la operación" (Calcagno 1985:59).

Para que este proceso tenga lugar tuvieron que darse ciertas condiciones, a saber:

1. Gran liquidez en los mercados financieros mundiales y baja demanda de créditos en los países desarrollados, lo cual favoreció que regiones como América Latina comenzaran a jugar un rol importante como tomadores de créditos.

2. Reforma financiera de 1977 en Argentina: completa liberalización del movimiento de capitales con el exterior.

3. El sector público tomó créditos destinados a la formación de reservas de divisas.

4. La tasa de interés interna era superior a la internacional. Esta brecha se acentuaba más ante expectativas de devaluación.

El ejemplo anterior permite ilustrar cómo las condiciones de la macro-estructura habilitan en algunos casos (e in-habilitan en otros) determinadas toma de decisiones. Los préstamos en dólares no habrían sido posibles si la liquidez de los mercados mundiales no hubiese sido abundante; sin la reforma financiera el flujo de capitales que se movía libremente entre Argentina y el resto del mundo no habría tenido lugar; el diferencial entre las tasas de interés interna e internacional fue quizás el mayor aliciente para emprender las maniobras especulativas; si el BCRA no hubiese tomado préstamos para la formación de reservas, la conversión de pesos a dólares se hubiese dificultado.

Como se puede apreciar, las relaciones que se dan entre las variables y/o entidades económicas es producto de las acciones de las personas y del contexto que condiciona a las mismas en la toma de decisiones. Esto es lo que da lugar a los procesos socioeconómicos. Sin embargo, tanto el contexto como 
las acciones son muy volátiles, lo que hace que éstos no pertenezcan a ninguna de las dos clases de procesos que se reconocen en Glennan (2002): no son ni frágiles ni robustos. No son secuencias frágiles o singulares, pues poseen una estructura característica que se puede mantener a lo largo del tiempo. Pero esa estructura no es tan "estable" como los procesos que Glennan denomina "robustos", pues diversos desenlaces futuros son plausibles. Son así algo diferente, de carácter intermedio, entre la fragilidad y la robustez extremas.

Tomemos como ejemplo de la representación de un proceso socioeconómico el "efecto Keynes". Se trata de un proceso por el cual un aumento en la cantidad real de dinero conduce a un descenso en la tasa de interés, estimulando la inversión y en consecuencia el empleo y la producción. Ahora bien, es erróneo pensar que un cambio positivo en la cantidad real de dinero conducirá de manera invariante a un descenso en la tasa de interés, a un aumento en la inversión, y por consiguiente a un incremento en el nivel de empleo y de la renta nacional. Por el contrario, de acuerdo con el marco contextual y con las interpretaciones y expectativas que formen las personas, distintos serán los caminos que puedan tomar esta clase de procesos. Keynes ha sido explícito al respecto: "si bien puede esperarse que, ceteris paribus, un aumento en la cantidad de dinero reduzca la tasa de interés, esto no sucederá si las preferencias por la liquidez del público aumentan más que la cantidad de dinero; y mientras que puede esperarse que, ceteris paribus, un descenso en la tasa de interés aumente el volumen de la inversión, esto no ocurrirá si la curva de la eficiencia marginal del capital baja con mayor rapidez que la tasa de interés; y mientras es de suponer que, ceteris paribus, un aumento en el volumen de la inversión haga subir la ocupación, esto puede no suceder si la propensión marginal a consumir va en descenso" (1936:150, énfasis en original).

Este ejemplo permite mostrar que los procesos económicos no responden de manera adecuada a la lógica de la invarianza y de los mecanismos. Por el contrario, se ajustan mejor a la lógica de los "árboles de posibilidades" o "resultados de final abierto": dado un acontecimiento determinado (inflación, aumento del gasto público, huelga gremial, etc.), existen diferentes caminos o alternativas. Cualquiera de éstos es en principio plausible. Su acontecimiento o no dependerá de cómo las personas formen sus expectativas en ese momento, del marco cultural, institucional, etc. En este sentido, no parece apropiado hacer afirmaciones del tipo "existe un mecanismo por el cual la oferta monetaria provoca cambios en la renta nacional". No hay una fuerza causal que induzca a las personas a demandar mayor cantidad de activos financieros cada vez que la cantidad real de dinero aumente en una economía. Las personas pueden volcar estos incrementos a la compra de bienes y servicios o al atesoramiento. Las acciones no están -por decirlo de alguna manera- "predeterminadas", sino que dependen fuertemente de las condiciones del contexto, de cómo formen sus expectativas, etc.

Los modelos explicativos examinados en el presente trabajo están pensados para aquellos sistemas donde predomina la estabilidad, en el sentido de que las relaciones causales no suelen presentar alteraciones sistemáticas. Empero, en un sistema donde lo que prevalecen son los "árboles de posibilidades" o donde las relaciones causales y los parámetros son de "cambio rápido", estos modelos son infructuosos, ya que es precisamente la apelación a relaciones invariantes, leyes y/o mecanismos el requisito más importante para lograr profundidad explicativa.

Contrario a estos modelos, se argumentará aquí que el explanans o argumento explicativo de un proceso socioeconómico va a estar conformado por dos clases de enunciados: (1) las acciones de las personas y (2) los factores contextuales. 
Es sencillamente imposible explicar un fenómeno social sin hacer referencia a la acción humana. Por ejemplo, para entender por qué baja la tasa de interés ante una subida en la cantidad real de dinero, uno debe explicitar qué decisiones estarían tomando las personas. Sin embargo, estas acciones no se comprenden en su totalidad si no se articulan con las condiciones de la macro-estructura y con el acervo de señales que reciben e interpretan los agentes, esto es, con los factores contextuales. Nótese que en esta clase de argumentos no hay ningún compromiso con el comportamiento estable o regular. Las acciones de las personas pueden ser únicas para ese momento, al igual los factores contextuales que las acompañan. Sin embargo, el argumento que se obtenga de ello servirá perfectamente para propósitos explicativos.

El argumento que se pretende defender aquí es una crítica a los enfoques de explicación que exigen estabilidad para catalogar a dicha explicación como "profunda" o "científica". No obstante, esto no implica una crítica per se a los modelos que pretenden estabilidad. Estos modelos resultan ser muy útiles para explicar fenómenos naturales, o hasta incluso fenómenos sociales donde prevalece el funcionamiento de mecanismos psicológicos (nótese por ejemplo el éxito de varios modelos de Behavioral Economics). Sin embargo, no van a ser adecuados para explicar aquellos fenómenos que no son producto de un acervo de factores invariantes.

Por consiguiente, si bien se reconoce que las regularidades y los mecanismos pueden facilitar determinadas explicaciones, deben tenerse presente dos cosas. En primer lugar, que la apelación a regularidades y/o mecanismos no es ni necesaria ni suficiente para proporcionar una explicación adecuada de algún fenómeno socioeconómico. No es suficiente, ya que si no se dispone de las condiciones antecedentes pertinentes no se podrá explicar ningún hecho concreto (lo que el mismo Hempel reconoce). Tampoco es necesaria. Solo se requiere conocer qué factores contextuales sobresalieron y cómo han actuado las personas en el momento en que aconteció el fenómeno explanandum.

En segundo lugar, dada la lógica de los sistemas socioeconómicos examinada anteriormente, las regularidades y los mecanismos pueden ser posibles, pero su duración en el tiempo será mucho más corta que los fenómenos de la naturaleza. En cuyo caso, la investigación científica deberá abocarse tanto al descubrimiento de las razones que dieron lugar a la estabilidad de un proceso socioeconómico en un momento del tiempo, como de los motivos que provocaron su ruptura. Un buen ejemplo de ello es la curva de Phillips. En 1958 el economista William Phillips publicó un artículo titulado "La relación entre el desempleo y la tasa de variación de los salarios monetarios en el Reino Unido, 1861-1957", en el cual se establecía la existencia de una correlación negativa entre la tasa de desempleo y la inflación. Dos años más tarde, Samuelson y Solow encontraron el mismo registro estadístico para Estados Unidos entre 1900 y 1960. El argumento propuesto fue el siguiente: dados los precios esperados, que para los trabajadores son simplemente los precios del año anterior, una reducción del desempleo provoca una subida de los salarios nominales, la cual provoca, a su vez, una subida de los precios. Reuniendo todos estos pasos, una reducción del desempleo provoca una subida de los precios este año en comparación con los del año pasado, es decir, un aumento de la inflación.

No obstante, es bien conocido que esta regularidad se "quebró" a principios de 1970, el proceso inflacionario se acentuó durante estos años, lo cual modificó el modo en el que las personas formaban sus expectativas. Durante los periodos de inflación baja y poco persistente, tanto los trabajadores como las empresas solían no tener en cuenta la inflación pasada, suponiendo así que los precios en los próximos periodos no iban a diferir significativamente de los precios actuales. Pero en un marco de 
inflación sistemática, los agentes empezaron a suponer que, en los próximos periodos, los precios seguirían aumentando, haciendo que la relación inversa entre inflación y desempleo desaparezca. El ejemplo muestra que, de haber estabilidad en ciertas condiciones antecedentes $-v$. gr., expectativas de inflación baja- una regularidad puede emerger al nivel de los eventos. No obstante, ésta no es producto de fuerzas causales estables como los mecanismos o las leyes, sino de un conjunto de circunstancias específicas de validez temporal limitada. Cuando estas circunstancias o condiciones ya no se cumplan, la regularidad desaparecerá.

\section{Consideraciones finales}

Se ha procurado mostrar que los procesos socioeconómicos se adecúan mejor a la lógica de los árboles de posibilidades o resultados de final abierto que a la de los mecanismos, leyes y regularidades invariantes. Se ha reconocido en que en determinadas circunstancias las regularidades pueden emerger de los sistemas socioeconómicos. Sin embargo, éstas no sólo son excepcionales, sino que pueden "quebrarse" fácilmente (como es el caso del "efecto Keynes" o la "curva de Phillips"). Esto se debe a que los procesos socioeconómicos dependen de la macro-estructura y de las acciones de las personas, las cuales son muy volátiles. Se ha propuesto una salida alternativa a esta problemática: focalizar en los factores estructurales y en las decisiones de los agentes. Posiblemente haya algún conocimiento invariante en estas aserciones. Sin embargo, éste no es ni necesario ni suficiente para proporcionar un argumento con profundidad explicativa.

\section{Bibliografía}

Bunge, M. 2005. Buscar la filosofía en las ciencias sociales. Buenos Aires: Siglo XXI editores.

Calcagno, A. 1985. La perversa deuda argentina. Buenos Aires: Legasa.

Glennan, S. 2002. Rethinking mechanistic explanation. Philosophy of Science 69(3, supplement): 342353. doi: $10.1086 / 341857$

Hempel, C.G. 1979. La explicación científica. Estudios sobre la filosofía de la ciencia. Barcelona: Paidos.

Hempel, C.G. y Oppenheim, P. 1948. Studies in the logic of explanation. Philosophy of Science 15(2): 135175. jstor.org/stable/185169

Keynes, J.M. 1936. La teoría general de la ocupación, el interés y el dinero. Buenos Aires: Fondo de Cultura Económica.

Woodward, J. y Hitchcock, C. 2003. Explanatory generalizations, part I: a counterfactual account. Nous 37(1): 1-24. doi: 10.1111/1468-0068.00426.

Recibido el 15 Aug 2015

Aceptado el 25 Sep 2015 